12

\title{
Численное исследование температурных полей в плазмотроне постоянного тока
}

\author{
(С) А.И. Сайфутдинов ${ }^{1,2}$, Б.А. Тимеркаев ${ }^{3}$, А.Р. Ибрагимов ${ }^{1}$ \\ ${ }^{1}$ Казанский фредеральный университет, Казань, Россия \\ ${ }^{2}$ Санкт-Петербургский государственный университет, \\ Санкт-Петербург, Россия \\ ${ }^{3}$ Казанский национальный исследовательский технический университет \\ им. А.Н. Туполева - КАИ, Казань, Россия \\ E-mail: as.uav@bk.ru
}

Поступило в Редакцию 7 августа 2017 г.

В рамках численных экспериментов проведены исследования характеристик плазмотрона постоянного тока. Получены распределения температурных полей в канале плазмотрона, катоде и аноде. Показано, что температура рабочей поверхности катода превышает температуру плавления материала, из которого он состоит.

DOI: 10.21883/PJTF.2018.04.45641.17005

Плазмотроны различных типов находят широкое применение на протяжении многих лет. В настоящее время они используются в металлургии, для упрочнения поверхностей металлов, в процессах плазменного напыления материалов и получения мелкодисперсных порошков, для резки металлов и др. [1-3]. В связи с широким применением плазмотронов широк и диапазон предъявляемых к ним требований. Длительная работа плазмотрона напрямую связана с ресурсом работы отдельных его элементов, а именно электродов. Тепловое состояние поверхности, а также величина и характер эрозии термоэмиссионных катодов сильноточных плазменных систем определяют их работоспособность и ресурс работы. Взаимодействующая система электрод-прикатодная плазма является сложным для исследования объектом, характеризующимся сильной пространственно-временной неоднородностью. Неоднородности температурных полей поверхности термоэмиссионных катодов могут приводить к появлению зон локального перегрева, испарению материала, его разрушению в результате трещинообразования и другим 
явлениям и процессам, определяющим ресурс катода. По этой причине пространственно-временно́й анализ температурных полей на поверхности катодов сильноточных электрических дуг является важной исследовательской задачей. Следует отметить, что определению характеристик плазмотронов и их оптимизации посвящены многочисленные работы. При этом, несмотря на значительный прогресс, достигнутый в моделировании плазмотронов постоянного тока [4-8], температура внутренних поверхностей электродов задается постоянным значением, т. е. никак не учитывается нагрев электродов за счет процессов, происходящих на их границе с дуговой плазмой.

Целью настоящей работы является численное моделирование основных характеристик плазмотрона, в том числе и самосогласованного определения распределения температурных полей в электродах. Модель основана на системе уравнений Навье-Стокса, которая включает в себя уравнение неразрывности, уравнения движения и уравнение переноса тепла в плазменной струе, дополненной системой уравнений Максвелла для электрического и магнитного полей и дифференциального закона Ома, а также уравнениями теплового баланса для металлических катода и анода:

$$
\begin{gathered}
\frac{\partial \rho}{\partial t}+\nabla(\rho \mathbf{V})=0 \\
\rho\left(\frac{\partial \mathbf{V}}{\partial t}+\mathbf{V} \cdot \nabla \mathbf{V}\right)=\mathbf{j} \times \mathbf{B}-\nabla\left[P+\frac{2}{3} \mu(\nabla \cdot \mathbf{V})\right]+2 \nabla(\mu S), \\
\rho c_{p}\left(\frac{\partial T}{\partial t}+\mathbf{V} \cdot \nabla T\right)-\frac{d P}{d t}=\nabla\left(\lambda_{2} \nabla T\right)+\mathbf{j} \times \mathbf{E}+\frac{5 k_{\mathrm{B}}}{2 e} \mathbf{j} \cdot \nabla T-Q_{r a d} \\
\nabla(\sigma \nabla \varphi)=0, \quad \mathbf{E}=-\nabla \varphi, \mathbf{j}=\sigma \mathbf{E}, \quad \nabla^{2} \mathbf{A}=-\mu_{0} \mathbf{j}, \quad \mathbf{B}=\nabla \times \mathbf{A}, \\
\rho_{c, a} c_{p c, p a}\left(\frac{\partial T_{c, a}}{\partial t}\right)=\nabla\left(\lambda_{c, a} \cdot \nabla T_{c, a}\right) .
\end{gathered}
$$

Здесь $\rho, \rho_{c}, \rho_{a}$ - плотность газа, материала катода и анода соответственно, $t$ - время, $\mathbf{V}$ - скорость, $\mathbf{B}$ - вектор магнитной индукции, $P$ - давление газа, $\mu$ - динамическая вязкость, $c_{p}, c_{p c}, c_{p a}-$ соответственно теплоемкость рабочего газа, материала катода и анода при постоянном давлении, $T, T_{c}, T_{a}$ - температура в плазменном канале, катоде и аноде соответственно, $\mathbf{E}$ - напряженность электрического поля, $\lambda, \lambda_{c}, \lambda_{a}-$ теплопроводность газа, материала катода и анода

Письма в ЖТФ, 2018, том 44, вып. 4 
Граничные условия

\begin{tabular}{|c|c|}
\hline Область & Граничные условия \\
\hline Вход в канал плазмотрона & $T=T_{0}, \int_{\partial \Omega} \rho(\mathbf{V} \cdot \mathbf{n}) d_{b c} d S=m, \mathbf{j} \cdot \mathbf{n}=0, \mathbf{A} \times \mathbf{n}=0$ \\
\hline Рабочая поверхность катода & $T=T_{c}, \mathbf{V}=0, \mathbf{A} \times \mathbf{n}=0,(8)$ \\
\hline Рабочая поверхность анода & $T=T_{a}, \mathbf{V}=0, \mathbf{A} \times \mathbf{n}=0,(7)$ \\
\hline $\begin{array}{l}\text { Внешние границы плазменной } \\
\text { области }\end{array}$ & $\nabla T=0, P=P_{0}, \mathbf{j} \cdot \mathbf{n}=0, \mathbf{A} \times \mathbf{n}=0$ \\
\hline $\begin{array}{l}\text { Внешние границы катода } \\
\text { и анода }\end{array}$ & $T=T_{0},\left.\int_{\partial \Omega} \mathbf{j} \cdot \mathbf{n} d S\right|_{c}=I_{0},\left.\varphi\right|_{a}=0$ \\
\hline
\end{tabular}

Примечание. $\partial \Omega-$ сечение канала, через которое подается плазмообразующий газ, $d_{b c}$ - граница входа плазмообразующего газа, $m$ - массовый расход плазмобразующего газа, $d S$ - элементарное сечение канала, через которое массовый расход постоянен, $\mathbf{n}-$ вектор нормали, $P=P_{0}$ - давление газа на выходе, $I_{0}-$ ток дуги плазмотрона.

соответственно, $\sigma$ - удельная проводимость, $\varphi$ - электрический потенциал, $\mathbf{A}$ - векторный потенциал магнитного поля, $\mu_{0}$ - магнитная проницаемость вакуума, $\mathbf{j}$ - плотность полного тока, $k_{\mathrm{B}}$ - постоянная Больцмана, $e-$ заряд электрона, $Q_{r a d}-$ удельная мощность излучения, зависящая от температуры.

Вычислительная область состояла из трех доменов: катод, анод и плазменный канал. Граничные условия приведены в таблице.

Рассмотрим более подробно условия на границах раздела между дуговой плазмой и электродами.

1. На границе между дуговой плазмой и анодом ставится следующее условие [5]:

$$
\left[-\left(\lambda_{a} \cdot \nabla T_{a} \cdot(-\mathbf{n})\right)\right]_{\text {anode }}-[-(\lambda \cdot \nabla T(-\mathbf{n}))]_{\text {plasma }}=|\mathbf{j} \cdot \mathbf{n}| \phi_{a}-\varepsilon \sigma_{\mathrm{B}} T^{4}
$$

Оно показывает, что нормальный поток тепла на анод включает поток тепла за счет теплопроводности из плазмы, поток тепла за счет бомбардировки электронами и охлаждение за счет излучения твердого тела. Здесь $\phi_{a}$ - работа выхода электрона из металлического анода, $\sigma_{\mathrm{B}}$ - постоянная Стефана-Больцмана.

2. Вдоль границы дуговая плазма-катод нормальный разрыв теплового потока выражается следующим образом: учитывается перенос

Письма в ЖТФ, 2018, том 44, вып. 4 


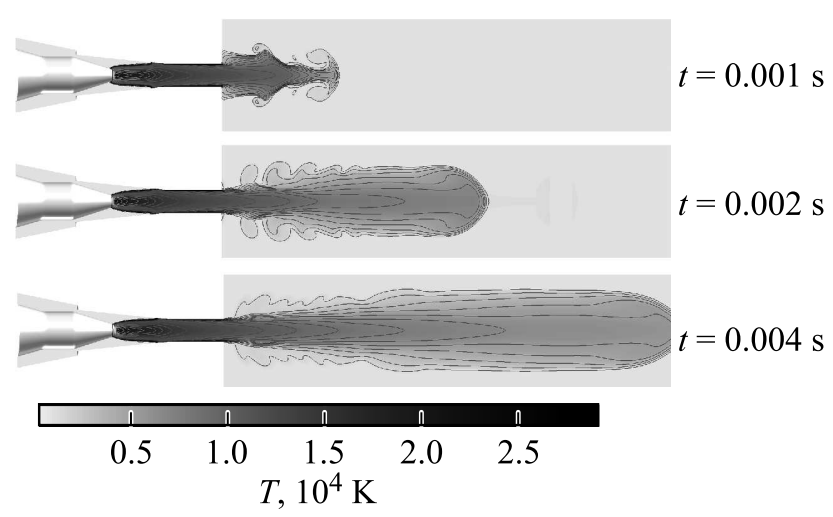

Рис. 1. Динамика формирования температурного поля струи плазмотрона.

тепла из дуги на катод, нагрев за счет потока ионов, охлаждение за счет выхода электронов благодаря термоэлектронной эмиссии и охлаждение за счет излучения твердого тела, определяемого по закону Стефана-Больцмана:

$\left[-\left(\lambda_{c} \cdot \nabla T_{c} \cdot(-\mathbf{n})\right)\right]_{\text {cathode }}-[-(\lambda \cdot \nabla T \cdot(-\mathbf{n}))]_{\text {plasma }}=j_{i} \varphi_{i}-j_{e} \phi_{c}-\varepsilon \sigma_{\mathrm{B}} T^{4}$

где $\varphi_{i}$ и $\phi_{c}$ - потенциал ионизации буферного газа и эффективная работа выхода материала катода соответственно. Ионный $j_{i}$ и электронный $j_{e}$ токи определяются как

$j_{e}=\left\{\begin{array}{ll}j_{r}, & \left(|\mathbf{j} \cdot \mathbf{n}|-j_{r}\right)>0, \\ |\mathbf{j} \cdot \mathbf{n}|, & \left(|\mathbf{j} \cdot \mathbf{n}|-j_{r}\right)<0,\end{array} \quad j_{i}=|\mathbf{j} \cdot \mathbf{n}|-j_{e}, \quad j_{r}=A_{r} T^{2} \exp \left(\frac{-e \phi_{e}}{k_{\mathrm{B}} T}\right)\right.$,

где $A_{r}, \phi_{e}$ - константа Ричардсона-Дэшмана и эффективная функция работы выхода в случае термоэлектронной эмиссии соответственно.

Численные эксперименты были проведены на примере плазмотрона постоянного тока F4 с вольфрамовым катодом и медным анодом фирмы „Sulzer Metco“. В качестве рабочего газа использовался аргон. На рис. 1 представлены распределения температуры при расходе плазмообразующего газа $2 \mathrm{~g} / \mathrm{s}$ в различные моменты времени при разрядном токе дуги

Письма в ЖТФ, 2018, том 44, вып. 4 


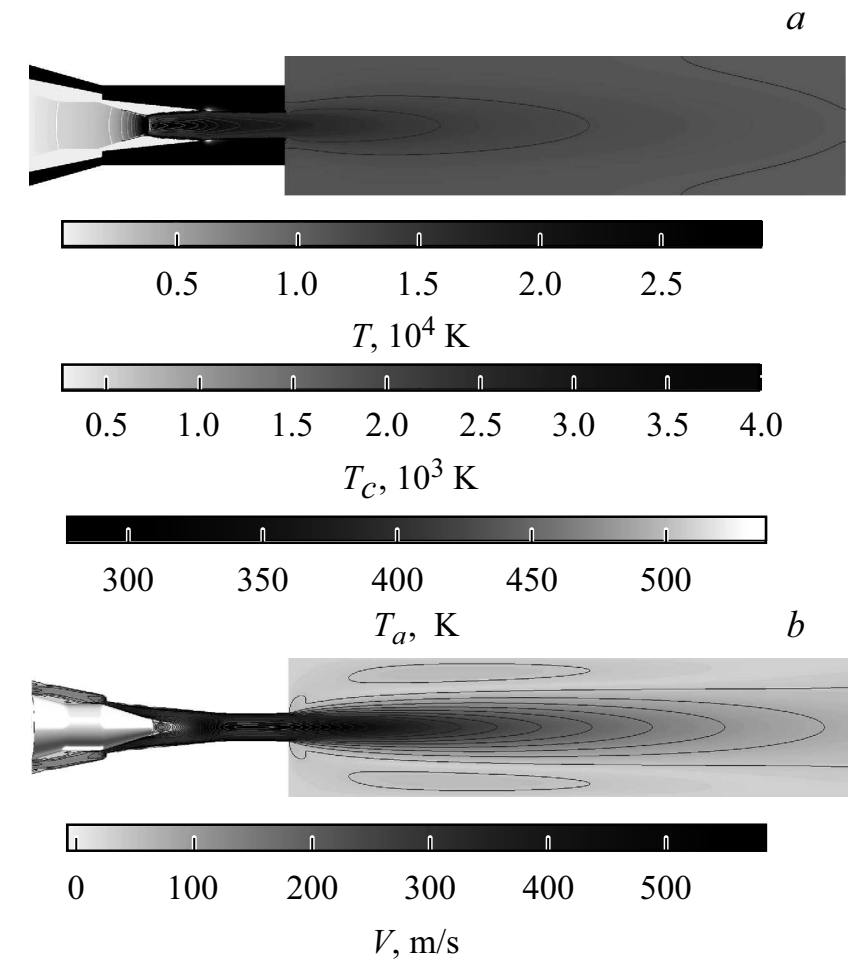

Рис. 2. Распределение температурных полей в плазмотроне $(a)$ и скорости плазменной струи $(b)$ при разрядном токе $300 \mathrm{~A}$ в момент времени $t=1 \mathrm{~s}$.

300 А. Видно, что время формирования установившегося плазменного потока составляет примерно $0.004 \mathrm{~s}$. На рис. 2 представлены распределения температурных полей в плазмотроне (в дуговом канале, катоде и аноде) при тех же условиях в момент времени 1 s. Из рисунка видно, что температура газа в плазменном канале плазмотрона достигает значений в $25000 \mathrm{~K}$, при этом температура рабочей поверхности вольфрамового катода превышает температуру плавления и составляет порядка $4000 \mathrm{~K}$, а температура поверхности медного анода в точке привязки дуги немного превосходит $500 \mathrm{~K}$. Следует отметить, что уже в момент

Письма в ЖТФ, 2018, том 44, вып. 4 
времени $0.3 \mathrm{~s}$ температура на рабочей поверхности катода достигает температуры плавления вольфрама.

Кроме того, обращает на себя внимание резкое изменение температурного поля по координате $z$ вблизи опорного пятна дуги. Так, при удалении от рабочей поверхности в глубь катода всего на $1 \mathrm{~mm}$ температура падает более чем на $800 \mathrm{~K}$, что объясняется хорошим охлаждением внешней стороны катода. При увеличении разрядного тока растет и температура рабочей поверхности катода. В частности, при токах 400, 500 и 600 А температура поверхности катода достигает 4200, 4400 и $4500 \mathrm{~K}$ соответственно. При этом глубина проплавления при разрядном токе 600 А составляет $1.8 \mathrm{~mm}$.

Таким образом, в работе сформулирована модель плазмотрона постоянного тока с учетом самосогласованного нагрева электродов. В рамках численных экспериментов впервые показано, что температура рабочей поверхности катода превышает температуру плавления вольфрама. Полученные результаты могут быть использованы при исследовании эрозии электродов в современных плазмотронах.

Работа поддержана грантом РФФИ 16-38-60187 мол_а_дк.

\section{Список литературы}

[1] Жуков М.Ф., Засыпкин И.М., Тимошевский А.Н., Михайлов Б.И., Десятков Г.А. Электродуговые генераторы термической плазмы. Т. 17. Низкотемпературная плазма. Новосибирск: Наука, 1999. 712 с.

[2] Глебов И.А., Рутберг Ф.Г. Мощные генераторы плазмы. М.: Энергоатомиздат, 1985. $264 \mathrm{c.}$

[3] Фролов В.Я., Иванов Д.В., Шибаев М.А. // Письма в ЖТФ. 2014. Т. 40. В. 16. C. 1-7.

[4] Chazelas C., Trelles J.P., Choquet I., Vardelle A. // Plasma Chem. Plasma Process. 2017. V. 37. N 3. P. 627-651.

[5] Chang C.H., Ramshaw J.D. // Plasma Chem. Plasma Process. 1993. V. 13. N 2. P. $189-209$.

[6] Li H.P., Chen X. // Plasma Chem. Plasma Process. 2002. V. 22. N 1. P. 27-58.

[7] Ramachandran K., Kikukawa N., Nishiyama H. // Thin Solid Films. 2003. V. 435. N 1-2. P. 298-306.

[8] Trelles J.P., Chazelas C., Vardelle A., Heberlein J.V.R. // J. Thermal Spray Technol. 2009. V. 18. N 5/6. P. 728-752.

Письма в ЖТФ, 2018, том 44, вып. 4 